\title{
Monitoring the Maintainability of Deep Inspiration Phase with Cine Acquisition in Patients with Left-Sided Breast Carcinoma Receiving Radiotherapy
}

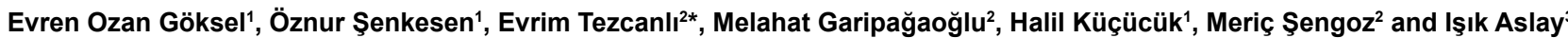

${ }^{1}$ Acibadem Kozyatagi Hospital, Department of Radiation Oncology, Istanbul, Turkey

${ }^{2}$ Acibadem University, Department of Radiation Oncology, Istanbul, Turkey

3/stanbul University, Oncology Institute, Capa Istanbul, Turkey

\begin{abstract}
Objectives: This prospective study aimed to investigate whether left-sided breast carcinoma patients could maintain deep inspiration (DI) during radiotherapy in order to reduce exposed heart volume.

Methods: Twenty-one patients with left breast carcinoma receiving adjuvant breast radiotherapy were included in this study. Breath hold time was monitored using the cine acquisition mode (CAM) of a linear accelerator during treatment in every fraction. Simulated $\mathrm{kV}$ and treatment field images were compared to determine whether the patient could achieve the same "breath holding level" (BHD). Maintenance of BHD during beam on time was referred as "maintenance of intrafraction breath holding level" (BHM), and chest wall displacement during each treatment was measured in order to verify the BHM. Furthermore, the relation between treatment time and patient compliance was studied.
\end{abstract}

Results: Sixteen patients were treated according to the deep inspiration breath hold (DIBH) protocol while 5 patients, excluded due to noncompliance, received their treatment without breath control. For the patients treated with DIBH protocol, maximum heart doses without breath control and DI phases were 3044 cGy and 1347 cGy, while mean heart doses were $348 \mathrm{cGy}$ and $239 \mathrm{cGy}$, respectively. Median BHD and BHM displacements were $0.2 \mathrm{~cm}$ $(0-0.86 \mathrm{~cm})$ and $0.07 \mathrm{~cm}(0-0.31 \mathrm{~cm})$, respectively. BHD differences were $<0.4 \mathrm{~cm}$ in all but 2 patients. Treatment time did not affect patient compliance.

Conclusions: DI phase was successful in reducing heart dose and $76.2 \%$ of the patients were eligible for DIBH. Treatment during moderate DI phase can be used for selected left-sided breast carcinoma patients and CAM could monitor safe execution.

Keywords: Radiotherapy; Breast carcinoma; Cardiac injury; Deep inspiration; Computerized tomography

\section{Introduction}

Adjuvant radiotherapy (RT) is one of the essential elements of breast conserving treatment and increases survival and local control after mastectomy in patients with $\mathrm{T} 3-4$ or any $\mathrm{N}+$ breast cancer $[1,2]$. However, some studies have reported lower survival rates in left-sided breast carcinoma due to radiation-related morbidity in comparison to that of right-sided breast carcinoma [3]. Detrimental side effects are not limited to high-dose radiation. Cardiac injury rates secondary to RT increase with radiation dose and elapsed time, especially 10-15 years after RT [4]. Long term follow up of atomic bomb survivors exposed to low dose radiation around $4 \mathrm{~Gy}$, showed that they had increased heart disease [5]. There are studies searching for better techniques, such as intensity-modulated (IMRT) and respiratory-gated (RP) radiation therapy, in order to decrease both the heart and lung doses and exposed volumes [6,7]. It has been demonstrated that RP can be used to achieve decreased cardiac doses by reducing the irradiated heart volume since increase in lung volume secondary to deep inspiration (DI) leads to breast displacement away from the heart [8,9] (Figure 1). Although it is recommended, RP treatment is not available in most centres. Treatment during DI can be used to achieve the same results. Computerized tomography (CT) -based studies have observed a reduction of the exposed heart volume when patients were irradiated during the deep inspiration breath hold (DIBH) [8-11]. However, treatment success is based on accurate implementation of the technique. Therefore, it is mandatory to ensure that the patient maintains the same magnitude of DI during treatment as attained during the CT planning scan. In our voluntary DIBH treatment protocol, chest wall displacement was used as a surrogate for the phase of respiration and patient set-up was corrected via megavoltage portal images and monitored by $2 \mathrm{D}$ fluoroscopy images. This prospective study evaluated the compliance and maintenance of voluntary DI phase using the 'cine acquisition' mode in patients with left breast carcinoma.

\section{Methods and Patients}

Twenty-one patients with stage pT1-2NoMo left breast carcinoma who had undergone breast conservative surgery (BCS) requiring adjuvant RT to breast only were asked to consent to participate in an institutional review board-approved protocol. Patients requiring

*Corresponding author: Evrim Tezcanli, Acibadem University, Radiation Oncology Department, Acibadem Kozyatagi Hospital, Inonu Cad., Okur Sok, No:20, Kozyatagi, Istanbul, Turkey, Tel: +90 21665884 36; E-mail: tezcanlievrim@gmail.com

Received January 26, 2013; Accepted March 26, 2013; Published March 30 2013

Citation: Göksel EO, Şenkesen Ö, Tezcanlı E, Garipağaoğlu M, Küçücük $H$, et al. (2013) Monitoring the Maintainability of Deep Inspiration Phase with Cine Acquisition in Patients with Left-Sided Breast Carcinoma Receiving Radiotherapy. J Nucl Med Radiat Ther 4: 141. doi:10.4172/2155-9619.1000141

Copyright: (C) 2013 Göksel EO, et al. This is an open-access article distributed under the terms of the Creative Commons Attribution License, which permits unrestricted use, distribution, and reproduction in any medium, provided the original author and source are credited. 


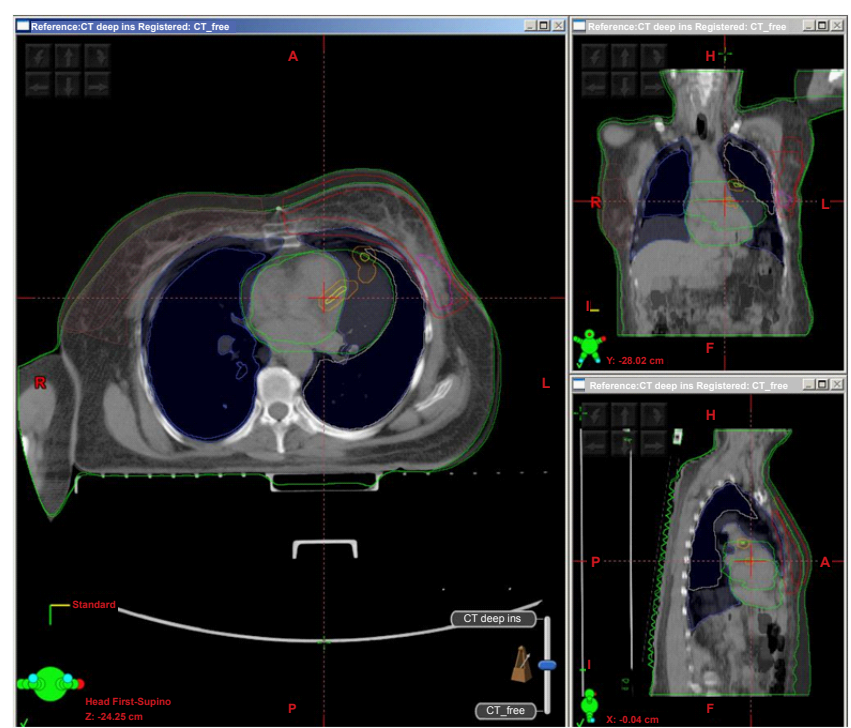

Figure 1: Heart and chest wall displacement secondary to respiratory motions differences between the FB and DI scans for a patient.

peripheral lymph node radiation were not included, given the fact that we cannot monitor chest wall displacement during respiration in regional lymph node areas. Left-sided, early stage breast cancer patients (pT1-2NoMo) that could maintain same breath hold level during DI for 30 seconds were eligible for the study. There were no limitations for age and co-morbid diseases. The DIBH protocol was initiated in our clinic in May 2009. This study includes 21 consecutive patients that were included in the DIBH protocol in order to reduce cardiotoxicity. The treating physician provided all patients with an oral explanation of the technique. All patients were physically trained for the breath-hold technique by a dedicated therapist and an adequate understanding of the procedure was ensured. Audiovisual equipments were not used for patient training. The patients' comprehension of the technique was verified with inspection of the diaphragm and chest wall movements for $30 \mathrm{~s}$ in the simulator via real time $\mathrm{kV}$ fluoroscopy before a CT scan was carried out. The mean absorbed dose to skin for fluoroscopy was $15 \mathrm{mGy}$ for $30 \mathrm{~s}$ but was subject to considerable variation with factors such as patient size and $\mathrm{kV}$ [12]. The patients' eligibility was also determined during this procedure before they were included in the study. All patients were positioned supine on a carbon fibre breast board with a fixed base accompanied by an adjustable tilt to ensure the sternum was in a horizontal position with the ipsilateral arm above the head. A body cast fabricated to immobilize the patient's shoulder was used to ensure daily set-up accuracy. All patients were scanned with a multi-detector 16 slice CT (Siemens Sensation 16 Erlangen, Germany) in the treatment position on a flat tabletop. Images were obtained as two different sets in a series, taken during free breathing (FB) and DI with 3-mm intervals. According to International Commission on Radiological Protection publication number 87, mean absorbed dose to breast was approximately $21 \mathrm{mGy}$ for each thorax CT scan [13]. Accuracy of the technique and the patients' ability to maintain DIBH were also verified with CT images. Images were transferred via network to the treatment planning system in DICOM III format. CT images taken during DI were registered to FB images, according to DICOM coordinates. ECLIPSE version 8.6 (Varian Inc., Palo Alto, USA) RT planning system was used for planning. The target (whole breast) and the organs at risk (OAR) [the lung, the heart, the LAD (left anterior descending artery), the contralateral breast] were delineated on the FB and DI series according to RTOG breast contouring recommendations with minor differences as previously published [9]. These differences included the contouring of LAD (left anterior descending artery) and the inclusion of whole pericardium to heart contouring. The initial treatment plans for each patient were constructed on the FB series using $3 \mathrm{D}$ conformal techniques.

In order to achieve sufficient dose distribution $6 \mathrm{MV}$ photon energy was preferred; however, $18 \mathrm{MV}$ was added when necessary. Treatment plans were obtained with either conformal planning using tangents or field-in-field IMRT technique. Inverse IMRT planning was not preferred given the long beam-on time and MLC blocking of fluoroscopy images that would disable the observation of chest wall movement via cine acquisition mode (CAM). Dynamic wedge was not used for the same reason. Treatment plans were evaluated based on our inhouse protocol, which considered target dose homogeneity and low OAR (the lung, the heart, and the LAD) doses, and were repeated using the DIBH series for patients when exceeded tolerance levels for heart doses. Patients were evaluated for DIBH technique when adequate target coverage and lower heart doses within tolerance levels were obtained with treatment plans that were constructed on DI images.

Our in-house protocol requires; (1) Daily fraction doses of 1.82 Gy given 5 times a week, (2) Whole breast dose to a total dose of 45- 50.4 Gy, (3) Boost dose of 10-16 Gy using 2 Gy/per fraction, (4) Total tumor bed dose was $60-66 \mathrm{~Gy}$, (5) $95 \%$ of clinical target volume (CTV) should receive $95 \%$ of the prescription dose; maximum dose within CTV is limited to $110 \%$, and this volume should be $<1 \%$, (6) the ipsilateral lung receiving $20 \mathrm{~Gy}<25 \%$, (7) as per institutional standards, heart volume receiving $35 \mathrm{~Gy}<1 \%(\mathrm{~V} 35<1 \%)$, and the mean heart dose $<3.5 \mathrm{~Gy},(8)$ the contralateral breast volume receiving $3 \mathrm{~Gy}<1 \%$, and the mean contralateral breast dose $<1 \mathrm{~Gy}$. Patients underwent a simulated run-through before treatment and fluoroscopy was carried out to ensure that the patients could maintain voluntary DIBH, $600 \mathrm{MU} / \mathrm{min}$ dose rate was chosen in order to reduce the total treatment time and DIBH period. A Varian Linear Accelerator (Varian Medical Systems, Clinac 2300 CD, Palo Alto, CA) with aS500 electronic portal imaging device (EPID) CAM was used for treatments. The Varian EPID (Varian Medical Systems, Palo Alto, CA) possesses a cine mode in which sequential images are acquired during the treatments and was used for tracking organ movement related to respiration [14,15]. Sequential images were obtained via cine mode during each treatment for each field and images were compared to simulation images acquired prior to treatment in the DI phase.

CAM was used to monitor maintenance of the DI phase during treatments through the treatment period of every fraction. Treatments were interrupted if any failure of DIBH was observed and restarted after corrections. In order to do so, treatment was restarted after a period of relaxation and a cine image was re-obtained in order to achieve an accurate measurement of breath hold level (BHD).

Treatment beams were used to obtain CAM images; therefore, no additional imaging dose was required. Varian aS500 EPID is capable of operating in cine mode at approximately 1 frame per 1.6 seconds.

Images were evaluated and compared digitally using image registration tools. Regions of interest consisted of the breast and lung volumes that contained soft tissue and air [16,17]. BHD defines a voluntary level of breath hold depending on each patient's breath holding ability. This level was determined based on planning CT images taken during voluntary DI. Same level of DI was tried to be 
achieved before starting each treatment. Achievement of same level was confirmed by measuring the lung distance at the center of the field. BHM defines maintenance of each patient's BHD during beam on time and it was evaluated by measuring the excursion of chest wall difference between the first and following CAM images during beam on time.

The level of DIBH was determined by comparing the chest wall position variations between CAM and initial $\mathrm{kV}$ images obtained in the simulator (Figure 2). Maintenance of intrafraction breath hold level (BHM) was quantified as the displacement of the chest wall between the first captured image and subsequent CAM images during beam on time (Figure 3).

\section{Results}

After evaluation of the planning CT series for both DIBH and FB phases, 2 of 21 patients were excluded as abdominal respiration was dominant for these patients. Given the limited respiratory movement and organ displacement, the DIBH did not have big influence on irradiated cardiac volumes and doses. The remaining patients underwent simulation with fluoroscopy to ensure the sustainability of DIBH. Although no significant differences were found for target doses between the DI and FB plans, the DI plans were better for the heart and LAD doses for the remaining 19 patients (Figure 4). In this group of patients maximum heart doses for FB and DI phases were 3044 cGy and 1347 cGy while mean heart doses were 348 cGy and 239 cGy respectively. However, 3 of the remaining 19 patients were excluded

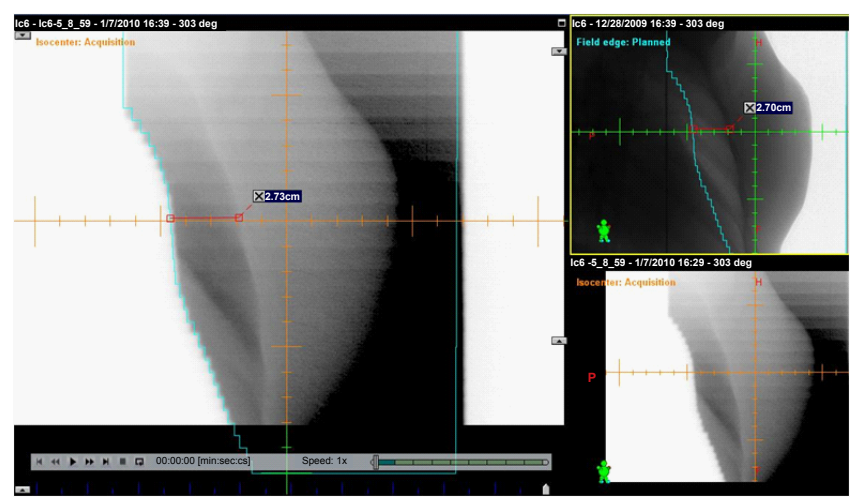

Figure 2: First cine acquisition and simulation images of treatment fields of the same fraction for a single patient.

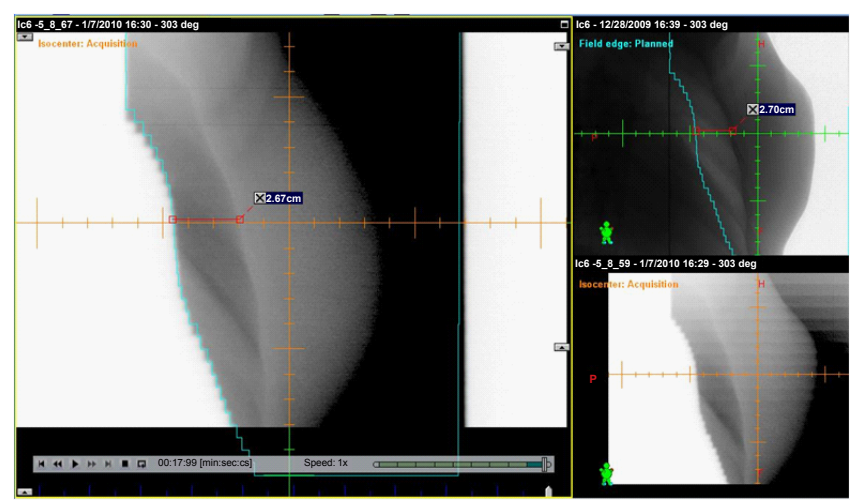

Figure 3: Last cine acquisition and simulation images of treatment fields of the same fraction for a single patient.

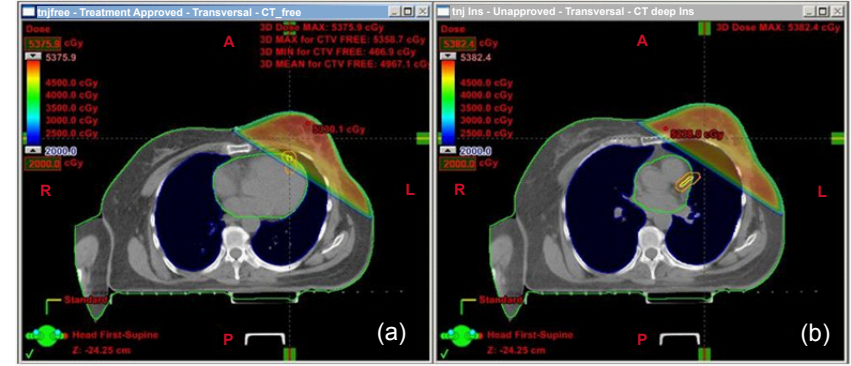

Figure 4: Comparison of dose distributions of (a) deep inspiration and (b) free breathing plans for a patient. Radiation exposed Left Anterior Descendent artery (LAD) (yellow color), LAD $+5 \mathrm{~mm}$ (orange color) and heart (green color).

as they could not accomplish DIBH and declared that they could not tolerate breath holding.

Consequently, 16 of 21 patients received treatment with the voluntary DIBH technique while the 5 excluded patients received treatment without breath control. No difficulties were observed in achieving and maintaining DIBH in the remaining 16 patients. Median age was 44 years old (range 32-58 years). Six patients had comorbid diseases such as history of lung tuberculosis, hypothyroidism secondary to thyroidectomy, cranial vessel aneurysm, myelodisplastic syndrome and panic disorder that was well tolerated during DI. None of the patients had dyspnea or lung disease that could cause respiratory insufficiency.

Two hundred and ten CAM images were evaluated. Image quality was found to be sufficient for evaluation. Median treatment time for each field was $11.97 \mathrm{~s}(6.65-26.98 \mathrm{~s})$. Median BHD and BHM displacements were $0.245 \mathrm{~cm}(0-0.86 \mathrm{~cm})$ and $0.07 \mathrm{~cm}(0-0.31 \mathrm{~cm})$, respectively. Measured displacements larger than $0.4 \mathrm{~cm}$ were observed in 2 fractions for 2 different patients and were measured as $0.86 \mathrm{~cm}$ and $0.84 \mathrm{~cm}$. These 2 patients were evaluated in details and discrepancies were measured. Each patient's median BHD and BHM displacements were $0.16 \mathrm{~cm}(0.01-0.86 \mathrm{~cm}), 0.03 \mathrm{~cm}(0-0.13 \mathrm{~cm})$, and $0.15 \mathrm{~cm}(0$ $0.84 \mathrm{~cm}), 0.05 \mathrm{~cm}(0.13-0.2 \mathrm{~cm})$, respectively. BHD differences were $<0.4 \mathrm{~cm}$ in all but these 2 patients for all fields and treatments. Patient compliance was sufficient for continuation of voluntary DIBH for treatments that lasted $<30 \mathrm{~s}$.

\section{Discussion}

Radiation exposure volumes and doses were found to be optimal for the heart, the LAD, and the lung when treated during DIBH compared to FB in all patients, which is compatible with the findings from previous studies $[18,19]$. According to present and previous study findings, patients with left-sided breast cancer might benefit from respiratory-gated treatment in the DI phase [8-11]. However, respiratory-gated treatment is not available in most centres. Our study demonstrated that the voluntary DIBH technique was easily applied to patients who comply with the technique; therefore, it is very important to determine the patient group for whom this treatment is applicable. For accurate treatment application, patients should be given adequate training prior to CT simulation and it should be verified that the patient could sustain the same phase of deep breathing during treatment. All patients had a good understanding of the technique. However, treating physician and dedicated technologist spent approximately 1 hour on each patient's training. Audiovisual coaching has been shown to increase reproducibility and compliance [20]. One could speculate that audiovisual equipment could be more time efficient. 
In the present study, abdominal respiration was dominant in 2 of $21(16.9 \%)$ patients therefore they were not able to hold their breath properly and were excluded from the protocol after CT simulation. Previously, Berson et al. [7] reported the similar problem that occurred in 12 out of 136 patients (9.5\%). Furthermore, three of the remaining 19 (14.2\%) patients could not maintain the DI phase during simulation of treatment plan and were also excluded. Likewise, compliance problem during DIBH was reported in the previous study where 8 out of $136(5.8 \%)$ patients could not tolerate the treatment during DI. In comparison to present study, both abdominal respiration dominancy and patient compliance problem rates were less. Present study included only patients with left breast carcinoma while Berson et al. [7] included patients with breast, lung, upper abdominal and mediastinal tumors. Ultimately, 5 of $21(23.8 \%)$ patients were not available for treatment during the moderate DI phase in this study while it was reported as 23 of 136 (16.9\%) patients in Berson et al. [7] study. Patient compliance is crucial for treatment success and it should be ensured accurately. For that reason, monitoring throughout the entire procedure is vital for use of this particular technique. Radiation fields for breast carcinoma involve 2 densities, such as the breast and the lung, which are easily differentiated from each other given their different tissue densities. Therefore, cine acquisition is a useful method to determine if the DI phase is achieved and sustained during the treatment as mentioned previously $[18,19,21]$. In this study, both achievement of DI and intrafraction target motion related to respiratory movement could be monitored in all patients via CAM. Indeed, it was demonstrated that portal MV imaging could be successfully used to obtain intrafraction set-up accuracy in liver, lung, and prostate and breast irradiation [15-18,21]. Therefore, CAM could be safely used to obtain set-up accuracy and detect variations in treatment during moderate DI. It was concluded that patient compliance was adequate.

As previously shown, Real-time Position Management (RPM) -gated RT successfully reduces the heart and lung volume exposed to radiation $[8,9,15]$,therefore such a technology will help to maximize the therapeutic ratio. It is expected that usage of RPM-gated RT will burgeon for left breast carcinoma patients receiving whole breast RT. One could speculate that human user may not be accurately detecting the level of breath holding as automated RPM. Therefore, using RPM may be more accurate and reproducible than voluntary breath holding. RT during moderate DI could be considered for selected patients who can maintain the DI phase during treatment in centres that have no RPM technology but are equipped with a linear accelerator furnished CAM.

\section{Conclusion}

Treatment in the DI phase is successful in reducing the heart and lung exposed dose and is tolerable for most patients, with $76.2 \%$ of our patients successfully complying with the voluntary DIBH technique. $\mathrm{RT}$ during the moderate DI phase, monitored via CAM, could be safely used in left breast carcinoma patients receiving whole breast RT who could maintain breath hold during the DI phase.

\section{References}

1. Fisher B, Anderson S, Bryant J, Margolese RG, Deutsch M, et al. (2002) Twenty-year follow-up of a randomized trial comparing total mastectomy, lumpectomy, and lumpectomy plus irradiation for the treatment of invasive breast cancer. N Engl J Med 347: 1233-1241.

2. Ragaz J, Olivotto IA, Spinelli JJ, Phillips N, Jackson SM, et al. (2005) Locoregional radiation therapy in patients with high-risk breast cancer receiving adjuvant chemotherapy: 20-year results of the British Columbia randomized trial. J Natl Cancer Inst 97: 116-126.
3. Paszat LF, Vallis KA, Benk VM, Groome PA, Mackillop WJ, et al. (2007) A population-based case-cohort study of the risk of myocardial infarction following radiation therapy for breast cancer. Radiother Oncol 82: 294-300.

4. Darby SC, McGale P, Taylor CW, Peto R (2005) Long-term mortality from heart disease and lung cancer after radiotherapy for early breast cancer: prospective cohort study ofabout 300,000 women in US SEER cancer registries. Lance Oncol 6: 557-565.

5. Preston DL, Shimizu Y, Pierce DA, Suyama A, Mabuchi K (2003) Studies of mortality of atomic bomb survivors. Report 13: solid cancer and noncancer disease mortality:1950-1997. Radiat Res 160: 381-407.

6. Remouchamps VM, Vicini FA, Sharpe MB, Kestin LL, Martinez AA, et al. (2003) Significant reductions in heart and lung doses using deep inspiration breath holdwith active breathing control and intensity-modulated radiation therapy for patientstreated with locoregional breast irradiation. Int J Radiat Oncol Biol Phys 55: $392-406$

7. Berson AM, Emery R, Rodriguez L, Richards GM, Ng T, et al. (2004) Clinica Experience Using Respiratory Gated Radiation Therapy: Comparison of FreeBreathing and Breath-Hold Techniques. Int J Radiat Oncol Biol Phys 60: 419426.

8. Korreman SS, Pedersen AN, Nøttrup TJ, Specht L, Nyström H (2005) Breathing adapted radiotherapy for breast cancer: comparison of free breathing gating with the breath-hold technique. Radiother Oncol 76: 311-318.

9. Korreman SS, Pedersen AN, Aarup LR, Nøttrup TJ, Specht L, et al. (2006) Reduction of cardiac and pulmonary complication probabilities after breathing adapted radiotherapy for breast cancer. Int J Radiat Oncol Biol Phys 65: 13751380.

10. Stranzl H, Zurl B (2008) Postoperative irradiation of left-sided breast cancer patients and cardiac toxicity. Does deep inspiration breath-hold (DIBH) technique protect the heart? Strahlenther Onkol 184: 354-358.

11. Tezcanli EK, Goksel EO, Yildiz E, Garipagaoglu M, Senkesen O, et al. (2011) Does radiotherapy planning without breath control compensate intra-fraction heart and its compartments' movement? Breast Cancer Res Treat 126: 85-92.

12. IAEA Training Material on Radiation Protection in Diagnostic and Interventional adiology (2000) Radiation Protection in Diagnostic and Interventional Radiology. L16.2: Optimization of Protection in Fluoroscopy Managing Patient Dose in Computed Tomography. ICRP publication.

13. ICRP Publication 87 (2000) Managing Patient Dose in Computed Tomography Ann ICRP 30.

14. Berbeco RI, Mostafavi H, Sharp GC, Jiang SB (2005) Towards fluoroscopic respiratory gating for lung tumours without radiopaque markers. Phys Med Biol 50: $4481-4490$

15. Remouchamps VM, Huyskens DP, Mertens I, Destine M, Van Esch A, et al (2007) The use of magnetic sensors to monitor moderate deep inspiration breath hold during breast irradiation with dynamic MLC compensators. Radiother Oncol 82: 341-348

16. Berbeco RI, Hacker F, Ionascu D, Mamon HJ (2007) Clinical feasibility of using an EPID in CINE mode for image-guided verification of stereotactic body radiotherapy. Int J Radiat Oncol Biol Phys 69: 258-266.

17. Tang X, Lin T, Jiang S (2009) A feasibility study of treatment verification using EPID cine images for hypofractionated lung radiotherapy. Phys Med Biol 54 S1-8.

18. Sixel KE, Aznar MC, Ung YC (2001) Deep inspiration breath hold to reduce irradiated heart volume in breast cancer patients. Int J Radiat Oncol Biol Phys 49: 199-204.

19. Pedersen AN, Korreman S, Nyström H, Specht L (2004) Breathing adapted radiotherapy of breast cancer: reduction of cardiac and pulmonary doses using voluntary inspiration breath-hold. Radiother Oncol 72: 53-60.

20. Cerviño LI, Gupta S, Rose MA, Yashar C, Jiang SB (2009) Using surface imaging and visual coaching to improve the reproducibility and stability of deepinspiration breath hold for left-breast-cancer radiotherapy. Phys Med Biol 54 6853-6865.

21. Mao W, Hsu A, Riaz N, Lee L, Wiersma R, et al. (2009) Image-guided radiotherapy in near real time with intensity-modulated radiotherapy megavoltage treatment beam imaging. Int J Radiat Oncol Biol Phys 75: 603 610 . 